University of Wollongong

Research Online

Faculty of Engineering - Papers (Archive)

Faculty of Engineering and Information

Sciences

2005

\title{
Characterization of conducting-polymer-based bimorph vibration sensors
}

Weihua Li

University of Wollongong, weihuali@uow.edu.au

Geoffrey M. Spinks

University of Wollongong, gspinks@uow.edu.au

Lianbin Zhao

University of Wollongong

Yanzhe Wu

University of Wollongong, yanzhe@uow.edu.au

Dezhi Zhou

University of Wollongong

See next page for additional authors

Follow this and additional works at: https://ro.uow.edu.au/engpapers

Part of the Engineering Commons

https://ro.uow.edu.au/engpapers/2589

\section{Recommended Citation}

Li, Weihua; Spinks, Geoffrey M.; Zhao, Lianbin; Wu, Yanzhe; Zhou, Dezhi; and Wallace, G G.:

Characterization of conducting-polymer-based bimorph vibration sensors 2005, 301-308.

https://ro.uow.edu.au/engpapers/2589 
Authors

Weihua Li, Geoffrey M. Spinks, Lianbin Zhao, Yanzhe Wu, Dezhi Zhou, and G G. Wallace 


\title{
Characterization of Conducting Polymer Based Bimorph Vibration Sensors
}

\author{
W.H.Li ${ }^{\mathrm{a} *}$, G.M.Spinks ${ }^{\mathrm{a}}$,, L.B.Zhao ${ }^{\mathrm{a}}$, Y.Z.Wu ${ }^{\mathrm{b}}$, D.Zhou ${ }^{\mathrm{b}}$ and G.G.Wallace ${ }^{\mathrm{b}}$ \\ ${ }^{a}$ School of Mechanical, Materials and Mechatronic Engineering, \\ University of Wollongong, Wollongong, NSW 2522, Australia \\ ${ }^{\mathrm{b}}$ Intelligent Polymer Research Institute, University of Wollongong, \\ Wollongong, NSW 2522, Australia
}

\begin{abstract}
This paper presents theoretical and experimental investigation of mechanical-electrical properties of conducting polymers based bimorph sensors. A material parameter, $h_{C P}$, is proposed to represent linear relationship between induction charge and the applied external deformation. Based on this assumption, a constitutive equation for bimorph sensors under steady-state external loadings are constructed and then solved. Mechanical-electrical properties of bimorph sensors are experimentally studied using both vibration-amplitude sweep mode and frequency sweep mode. The material parameter $h_{C P}$, is identified by comparing theoretical analysis and experimental results. The applications of conducting polymers based bimorph sensors in smart structures are also discussed.
\end{abstract}

Keywords: conducting polymers, bimorph sensor, material parameter, induced voltage, vibration amplitude, frequency

\section{INTRODUCTION}

Conducting polymers have found applications in many fields, such as electronic devices ${ }^{1-3}$ and electromechanical devices $^{4-6}$. These materials exhibit responsive electro-chemical-mechanical properties that make them very useful for operation as sensors. Conducting polymers form selective layers in which the interaction between the analyte gas and the conducting matrix generates the primary changes of a physical parameter in the transduction mechanism. An example of this is the development of polypyrrole-based gas sensors ${ }^{7,8}$. The polymer resistance increases in the presence of a reducing gas such as ammonia, and decreases in the presence of an oxidizing gas such as nitrogen. The gases cause a change in the near surface charge carrier density by reacting with surface adsorbed oxygen irons. Another type of sensor developed is a biosensor ${ }^{9}$. These sensors utilize the ability of triiodide to oxidize polyacetylene as a means to measure glucose concentration. Glucose is oxidized with oxygen with the help of glucose oxidase. This produces hydrogen peroxide which oxidizes iodide ions to form triiodide ions. Hence, conductivity is proportional to the peroxide concentration which is proportional to the glucose concentration.

Similar to piezoelectric materials ${ }^{10}$, conducting polymers can be used to directly convert electrical energy into mechanical energy, and vice versa. This energy transduction utilizes the large changes in size during the doping and dedoping of many conducting polymers. An example of this is the development of bimorph sensors. Such sensors have futuristic applications in smart structures, such as vibration control, and active suspension systems on cars.

The Intelligent Polymer Research Institute (IPRI) at University of Wollongong, Australia, has done some work on conducting polymer bimorphs in terms of actuators and sensors ${ }^{11}$. However, a detailed modeling approach to practical applications is not available. This paper is an extension of our previous work. A material parameter is proposed to represent the linear relationship between induction charge and the applied external deformation. By analyzing the dynamic behavior of a bimorph conducting polymer sensor in connection with its electrical properties, the parameter will be identified and discussed.

\section{THEORETICAL ANALYSIS}

\subsection{General response of a vibration conducting polymer sensor with a proposed material constant $\boldsymbol{h}_{C P}$}

This study employs the conducting polymer bimorph in parallel connection as the basic structure of the sensor. The schematic diagram of the parallel bimorph is shown in Figure 1. Two conducting polymer plates, each of thickness $H$

*email: weihuali@uow.edu.au; phone: (61 2) 4221 3490; fax: (61 2) 42213101 
and length $L$ are put together. The thickness $H$ is set quite small compared with the length $L$. The width of the bimorph, $W$, is also assumed to be so much smaller than the length that flexural vibration of the sandwich beam can be considered negligible.

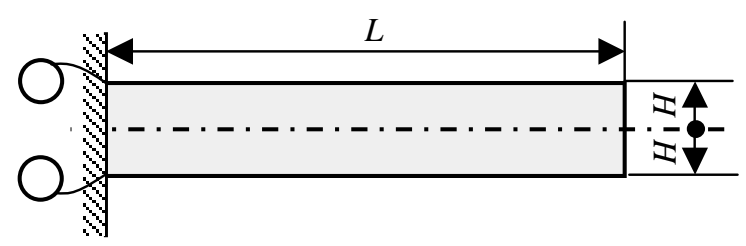

Figure 1. Schematic of the conducting polymer parallel connection bimorph

In order to analyze the electrical response of the structure, a constant force $P$ is applied to the free end of the bimorph cantilever beam. The bimorph deflects in proportion to the force $P$, as shown in Figure 2 . The vertical deflection causes the generation of electric voltage across the thickness of the beam due to conductivity. In parallel connection, the voltage across the thickness of the upper plate is the same as that of the lower plate. The magnitude of the generated voltage is a function of conducting polymer material, amount of the deflection, and the structure of bimorph.

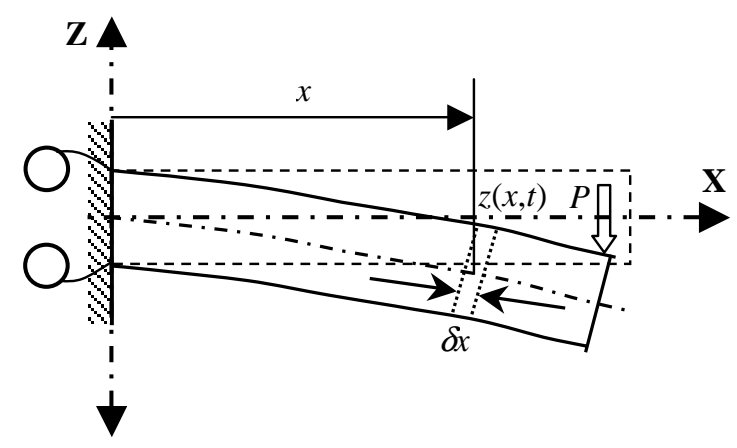

Figure 2 Deformation of the conducting polymer bimorph with a static force at its free end

In Figure 2, once the displacement $z(t)$ of the beam is given with respect to the external force $P$, the strain $s_{C P}(x, t)$ in the middle of the upper plate of the bimorph in thickness direction, i.e. $H / 2$, away from top surface of the beam, is given by $s_{C P}(x, t)=\frac{H / 2}{R(x, t)}$

where $R(x, t)$ is a radius of curvature of the beam at $x$ and is represented as

$R(x, t)=\left[\frac{d^{2} z(x, t)}{d x^{2}}\right]^{-1}$

Equation (2) is valid as long as $z(x, t)$ is small in comparison with the length of the beam. The deflection of the beam is considered linear along the thickness, and the deflection in the middle of the upper plate can represent the whole deformation of the cross-section of the beam. The $s_{C P}(x, t)$ in Equation (1) generates the electric field $E_{C P}$ with the following proposed constitutive equation

$E_{C P}(x)=h_{C P} s_{C P}(x, t)$

where $h_{C P}$ is our assumed conducting polymer constant causing generation of $E_{C P}$ in $Z$ direction with the strain $s_{C P}(x, t)$ in $X$ direction.

Combining Equations (1) to (3), the electric voltage at an arbitrary point $x$ of the upper conducting polymer plate becomes 
$V_{C P}(x, t)=H \cdot E_{C P}(x, t)=H \cdot h_{C P} \frac{H / 2}{R(x, t)}=h_{C P} \frac{H^{2}}{2} \cdot \frac{d^{2} z(x, t)}{d x^{2}}$

The measured voltage is not the voltage at such a specific point as Equation (4). The average voltage over the whole surface of the plate is given by the following integration equation

$V_{T}(x, t)=\frac{\int_{0}^{W} \int_{0}^{L} V_{C P}(x, t) d x d y}{A}=\frac{h_{C P} W H^{2}}{2 W L} \int_{0}^{L} \frac{d^{2} z(x, t)}{d x^{2}} d x=\frac{h_{C P} H^{2}}{2 L}\left[\left.\frac{d z(x, t)}{d x}\right|_{x=L}\right]$

where $A$ is planar area of the bimorph. Equation (5) is the proposed response equation of the conducting polymer vibration sensor. With this equation, the conducting polymer constant $h_{C P}$ can be identified if both the output voltage $V(t)$ and the deformation $z(x, t)$ with respect to an external force $P$ are given.

\subsection{Response of the sensor to sinusoidal forces}

The forced beam vibration theory is employed to analyze dynamic responses of the conducting polymers bimorph sensor. The system can be modeled as a cantilever Euler beam with an external force applied to its free end. Assume the free end displacement is a sinusoidal function with the amplitude $U_{0}$. Since the steady state response of the free end is $U_{0} \sin \omega t$, we can assume the free end load as $P_{0} \sin \omega t$. Thus, this problem can be represented as the free vibration equation

$\frac{\partial^{4} z}{\partial x^{4}}+\frac{1}{a^{2}} \frac{\partial^{2} z}{\partial t^{2}}=0$

where $a^{2}=\frac{E I}{\rho A^{\prime}}, E$ is Young's modulus, $I$ is the moment initial, $\rho$ is density, and $A^{\prime}$ is the cross section area of the beam.

The schematic diagram of the cantilever system is shown in Figure 3.

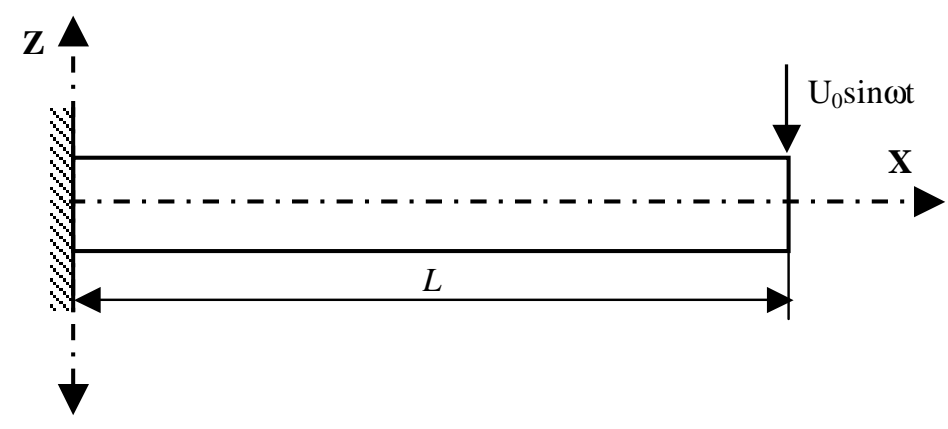

Figure 3 Schematic of a forced cantilever beam

The boundary conditions are listed below:

$z(0, t)=0, z(L, t)=U_{0} \sin \omega t, z^{\prime}(0, t)=0, E I z^{\prime \prime}(0, t)=0, E I z^{\prime \prime \prime}(L, t)=P_{0} \sin \omega t$

To solve Equation (6), assume the steady state response of the beam as

$z(x, t)=w(x) \sin \omega t$

Substituting Equation (8) into Equation (6), we get

$\frac{\partial^{4} w}{\partial x^{4}}-\beta^{4} w=0$

where $\beta^{4}=\frac{\omega^{2}}{a^{2}}$

The general solution of Equation (9) is

$w(x)=C_{1} \cos \beta x+C_{2} \sin \beta x+C_{3} \cosh \beta x+C_{4} \sinh \beta x$

The unknown constants $C_{1}, C_{2}, C_{3}$, and $C_{4}$ can be obtained by the boundary conditions as shown in Equation (7). 


$$
\begin{aligned}
& C_{1}=\frac{-\frac{1}{2}(\sin (\beta L)+\sinh (\beta L))}{-\cos (\beta L) \sinh (\beta L)+\cosh (\beta L) \sin (\beta L)} \cdot U_{0} \\
& C_{2}=\frac{\frac{1}{2}(\sin (\beta L)+\cosh (\beta L))}{-\cos (\beta L) \sinh (\beta L)+\cosh (\beta L) \sin (\beta L)} \cdot U_{0} \\
& C_{3}=\frac{\frac{1}{2}(\sin (\beta L)+\sinh (\beta L))}{-\cos (\beta L) \sinh (\beta L)+\cosh (\beta L) \sin (\beta L)} \cdot U_{0} \\
& C_{4}=\frac{-\frac{1}{2}(\sin (\beta L)+\cosh (\beta L))}{-\cos (\beta L) \sinh (\beta L)+\cosh (\beta L) \sin (\beta L)} \cdot U_{0}
\end{aligned}
$$

After getting the constants $\mathrm{C}_{1}, \mathrm{C}_{2}, \mathrm{C}_{3}$, and $\mathrm{C}_{4}$, the value of $\left.\frac{d w}{d x}\right|_{x=L}$ can be obtained

$$
\left.\frac{d w}{d x}\right|_{x=L}=\beta\left(-C_{1} \sin \beta L+C_{2} \cos \beta L+C_{3} \sinh \beta L+C_{4} \cosh \beta L\right)
$$

Substituting Equation (12) into Equation (5), the electrical output of the sensor is derived as

$V_{T}(t)=\frac{h_{C P} H^{2}}{2 L}\left[\left.\frac{d z(x, t)}{d x}\right|_{x=L}\right]=\frac{h_{C P} H^{2} \beta}{2 L}\left(-C_{1} \sin \beta L+C_{2} \cos \beta L+C_{3} \sinh \beta L+C_{4} \cosh \beta L\right) \sin \omega t$

With Equation (13), if the sensor dimensions, initial amplitude $U_{0}$ and the forcing frequency $\omega$ are provided, and the output voltage is measured as well, the proposed constant $h_{C P}$ can be identified. The following sections will test the validity of the model.

\section{EXPERIMENTAL}

\subsection{Bimorph sensor sample}

By electrochemical methods, the polypyrrole based conducting polymers bimorph sensors were prepared. The detailed synthesis processes are described in another paper ${ }^{12}$. The dimensions and physical properties of the bimorph sensor are listed in Table 1.

Table 1 Bimorph sensor properties

\begin{tabular}{|c|c|c|c|}
\hline $\mathbf{L}$ & $\mathbf{W}$ & $\mathbf{H}$ & $\mathbf{E}$ \\
$\mathrm{mm}$ & $\mathrm{mm}$ & $\mu \mathrm{m}$ & $\mathrm{GPa}$ \\
\hline 15 & 2 & 130 & 0.1 \\
\hline
\end{tabular}

\subsection{Experimental setup}

The schematic diagram of the testing system is shown in Figure 4. An advanced Dual-Mode Lever System is used to generate the different displacements as inputs and an A/D Instrument (MacLab/4e) is used for measurement of outputs of the voltage. Other equipments include Function Generator as input of Dual-Mode Lever System, PC with ChartViews software for analysis of the obtained data and auxiliary stand, clamps, steel stick and rubber band etc.

\subsection{Testing procedure}

In general, sensitivity and operating frequency range are two important factors in evaluating vibration sensors. Operating frequency means the flat frequency response region that is normally much lower than the fundamental resonant frequency of the sensor. The sinusoidal displacement excitation can be used to explore the properties of the sensitivity and operating frequency range of the polymer sensors and for the steady-state analysis of polymer sensor. For these purposes, both the amplitude sweep mode and the frequency sweep modes are employed. In other words, the measurement is to compare the sinusoidal loading input with the resultant voltage signal. 
(a) Amplitude Sweep Mode: The driving frequency is fixed at a given value, e.g. $5 \mathrm{~Hz}$, and the free end vibration amplitude $\left(U_{0}\right)$ is swept from $0.475 \mathrm{~mm}$ to $3.745 \mathrm{~mm}$ by adjusting the Dural-Model Level System, the induced voltages are recorded accordingly.

(b) Frequency Sweep Mode: The strain amplitude is fixed at a given value and the driving frequency $(\omega)$ is swept from $0.05 \mathrm{~Hz}$ to $10 \mathrm{~Hz}$. The frequency dependence of induced voltage will be investigated.

All the experiments are conducted at the room temperature.

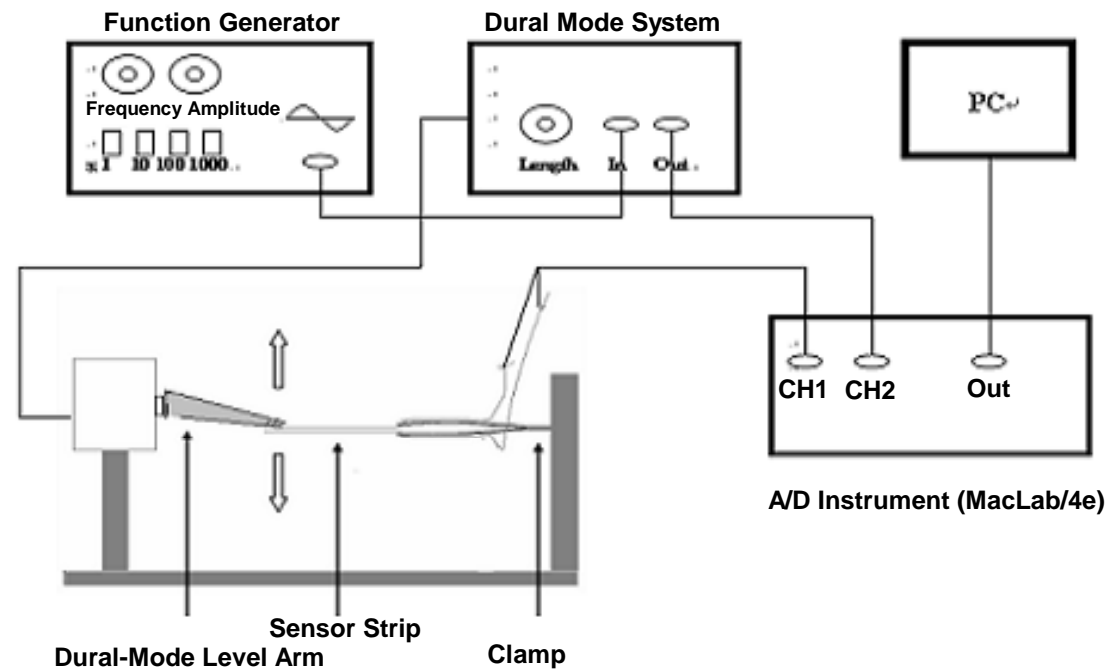

Figure 4 Schematic of the measuring system

\section{RESULTS AND DISCUSSION}

\subsection{Effect of vibration amplitude $\left(U_{o}\right)$}

Figure 5 shows the graph of resultant voltage as a function of free end amplitude at the constant driving frequency of 5 Hz. From this figure, it can be seen that as vibration amplitude increases, the induced voltage increases steadily, which agrees well with many reports in the literature. Due to the limitation of the experimental system, we cannot further increase the amplitude. In this study, there is no saturation observed.

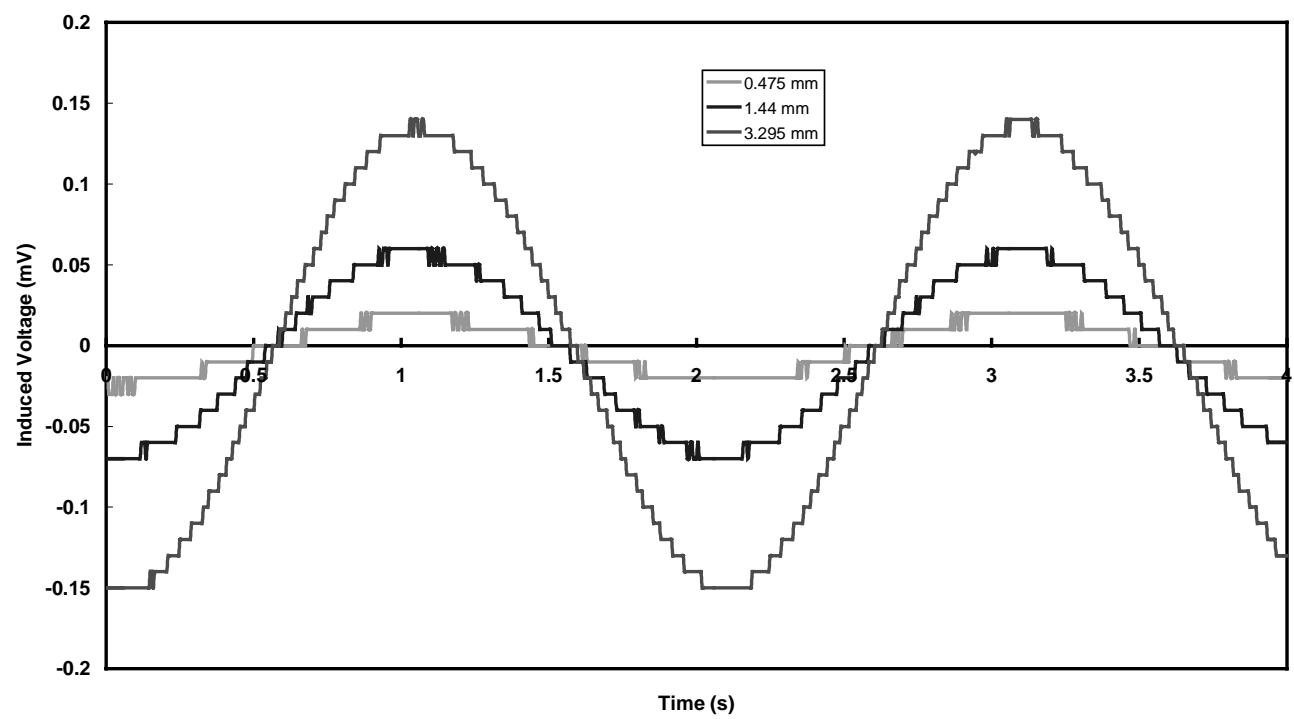

Figure 5 Induced voltage versus free end amplitude $\left(\mathrm{U}_{0}=0.475 \mathrm{~mm}, 1.44 \mathrm{~mm}, 3.295 \mathrm{~mm}\right)$ 


\subsection{Effect of driving frequency}

At a constant free end vibration amplitude $U_{0}=2 \mathrm{~mm}$, the frequency dependence of induced voltage is shown in Figure 6 . In Figure 6(a), the induced voltages are presented at $10,1,0.5,0.4,0.3,0.2,0.1$, and $0.05 \mathrm{~Hz}$ respectively from left to right.

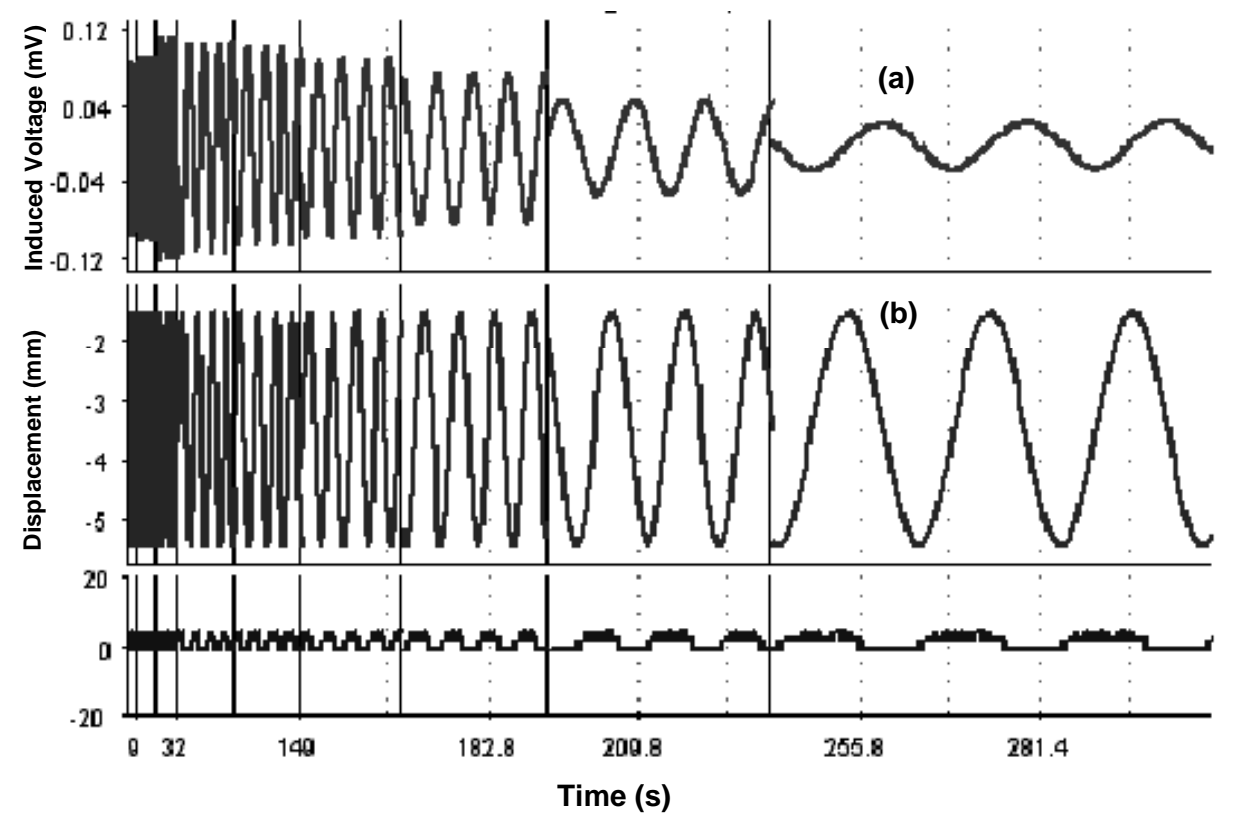

Figure 6 Frequency dependence of induced voltage (a) at (b) a constant vibration amplitude $U_{0}=2 \mathrm{~mm}$

For convenient analysis, the frequency dependence of induced voltage is summarized and shown in Figure 7. As can be seen from this figure, the induced voltage shows an increasing trend with frequency until a critical frequency is reached. Beyond this critical frequency, the induced voltage decreases slightly as the frequency is further increased. This result is quite different from the amplitude dependence of induced voltage. The mechanism as well as physical explanation is discussed elsewhere ${ }^{12}$.

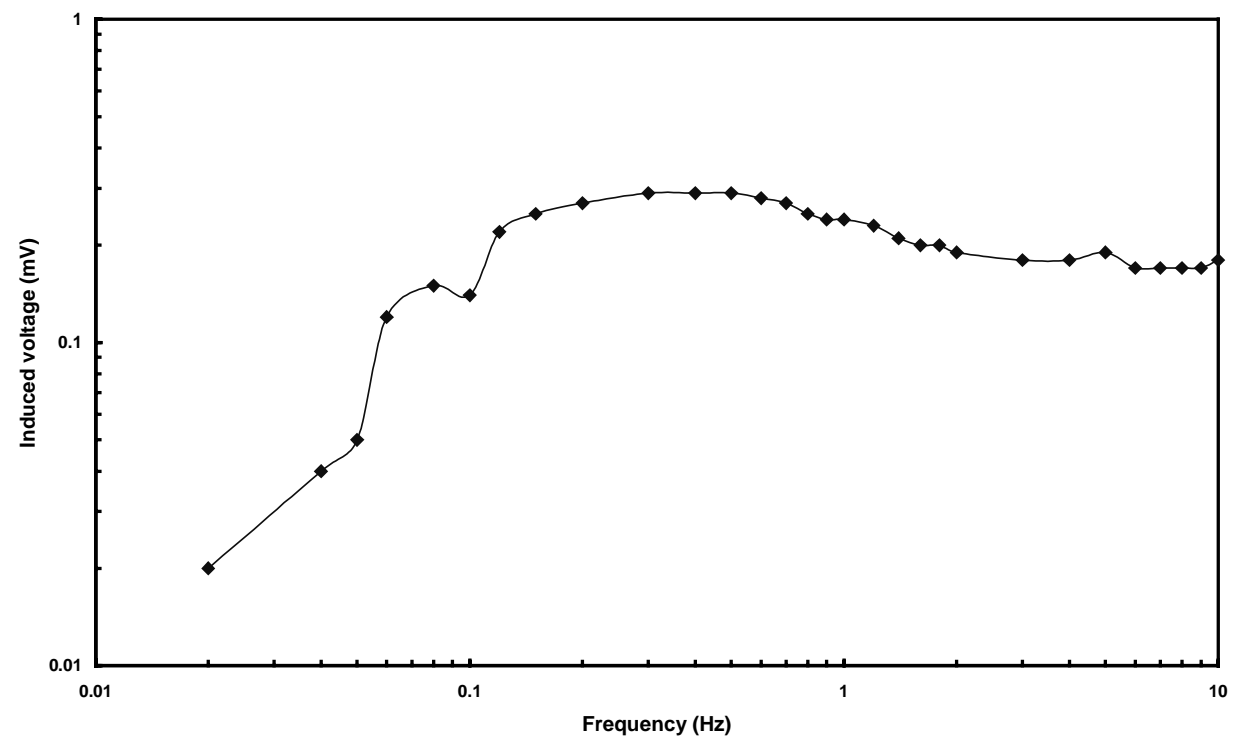

Figure 7 Induced voltages versus frequency at a constant vibration amplitude 


\subsection{Analysis of bimorph sensors using $h_{C P}$}

As discussed in Section 3, an induced constant $h_{C P}$ is defined and introduced to help represent material properties. Based on this model as shown in Equation (13), the induced voltage amplitude, $V_{T o}$ is represented as

$V_{T 0}=\frac{h_{C P} H^{2} \beta}{2 L}\left(-C_{1} \sin \beta L+C_{2} \cos \beta L+C_{3} \sinh \beta L+C_{4} \cosh \beta L\right)$

For a given bimorph sensor, the dimensions and material properties are fixed. Thus the parameters $H, L$, and $\beta$ are fixed as well. All the parameters $\mathrm{C}_{1}, \mathrm{C}_{2}, \mathrm{C}_{3}$, and $\mathrm{C}_{4}$ are all only proportional to vibration amplitude $U_{o}$. The relationship of $\mathrm{V}_{\mathrm{TO}}$ versus $U_{o}$ is shown in Figure 8. All the data are taken from the experimental results, some of which are presented in Figure 5.

Obviously, the induced voltage shows a perfectly linear trend with vibration amplitude. This finding justified our linear assumption. In other words, our proposed constant $h_{C P}$ is indeed independent of vibration amplitude and could be regarded as a parameter invariant to some extent. Due to the limitation of experimental conditions, we cannot get the results for large vibration amplitudes. There should reasonably exist a critical vibration amplitude, above which the linear assumption, $V_{T o} \propto U_{0}$, cannot be valid any more. Even though this study doesn't cover wide ranges of vibration amplitudes, both the experimental and analytical results would provide good guidance to design practical mechanical devices. For example, if the conducting polymer based devices operate at small amplitude conditions, these results will be very useful.

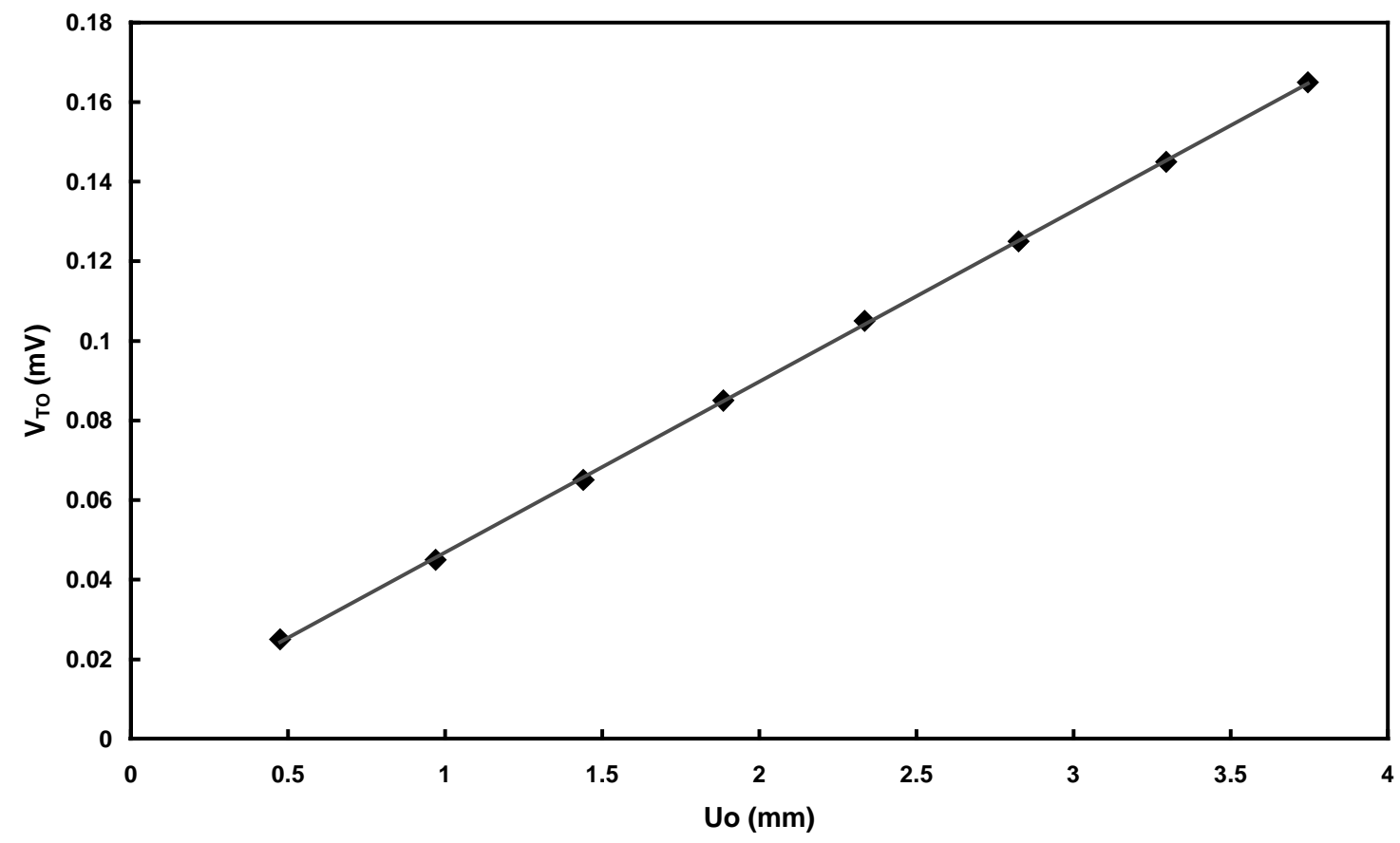

Figure 8 Induced voltage amplitude, $V_{T o}$, versus vibration amplitude, $U_{o}$,

In addition, this model doesn't consider amplitude-frequency coupling effect. Compared with amplitude influence, the frequency effect seems to be more complex. Even at very low frequencies $(<1 \mathrm{~Hz})$, the induced voltage shows obvious nonlinearity, as shown in Figure 7. It is crucial to find a comprehensive and accurate model to describe conducting polymer behaviors by considering not only vibration amplitude and frequency but also some other influencing factors. 


\section{CONCLUSIONS}

The aim of this paper was to characterize the electrical-mechanical transduction occurring in conducting polymer based bimorph sensors. The main findings are summarized as follows:

(1) A material parameter, $h_{C P}$, based on a linear assumption between the induced voltage and vibration amplitude, is proposed. With the reference of forced cantilever beam theory, the constitutive equations of bimorph sensors considering the material parameter $h_{c p}$ are derived. The steady-state responses of bimorph sensors under sinusoidal loading at the free end are derived. The analytical results indicate the induced voltage of conducting polymer bimorph sensors is proportional to the value $h_{c p}$, while sensor dimensions and other material properties, like Young's modulus, also influence the results. The parameter is identified by comparing experimental results and modeling analysis.

(2) The effects of both vibration amplitude and frequency on the induced voltage are experimentally studied by using vibration amplitude sweep mode and frequency sweep mode. At small amplitude ranges, the induced voltage increases steadily with the increment of vibration amplitude; Frequency dependence of induced voltage is quite complex. The induced voltage shows an increasing trend until the frequency reaches a critical value. Above the critical value, it decreases steadily.

(3) Comparison between theoretical analysis and experimental results using amplitude sweep mode justified that the proposed linear assumption. At small amplitude ranges, the induced voltage is proportional to vibration amplitudes. Such results would provide good guidances to design and develop conducting polymers based smart structures.

\section{REFERENCES}

1. M.K.Andrews, M.L.Jansen, G.M.Spinks, D.Zhou, and G.G.Wallace, "An integrated electrochemical sensor-actuator system," Sensors \& Actuators A, 114, pp. 65-72, 2004.

2. Han G., and G.Shi, "Conducting polymer electrochemical actuator made of high-strength three-layered composite films of polythiophene and polypyrrole," Sensors \& Actuators B, 99, pp. 525-531, 2004.

3. E.Smella, M.Kallenbach, J.Holdenried, "Electrochemically driven polypyrrole bilayers for moving and positioning bulk micromachined silicon plates," J. Microelectromechanical Systems, 8, pp.373-383, 1995.

4. Q.Pei, O.Inganas, "Conjugated polymers and the bending cantilever method: electrical muscles and smart devices," Adv.Mater., 4, pp. 277-278, 1992.

5. K.Kaneeto, M.Maneko, Y.Min, A.G.MacDiarmid, "Artificial muscles electromechanical actuators using polyaniline films," Synth. Met.., 71, pp. 91-93, 1995.

6. M.R.Gandhi, P.Murray, G.M.Spinks, G.G.Wallace, "Mechanism of electromechanical actuation in polypyrrole," Synth. Met., 73, pp. 247-256, 1995.

7. K.M.Lee, J.B.Yu, H.K.Jun, J.O.Lim, D.D.Lee, H.G.Byun, and J.S.Huh, "Volatile organic gas recognition using conducting polymer sensor array," Mater. Sci. Forum, 439, 344-351, 2003.

8. S.H. Hosseini, and A.A. Entezami, "Preparation and characterization of polyaniline blends with polyvinyl acetate, polystyrene and polyvinyl chloride for toxic gas sensors," Polymers for Advanced Technologies, 12, 482-493, 2001

9. M.El Kaoutit, D.Bouchta, H. Zejli, N. Izaoumen, K.R.Temsamani, "A simple conducting polymer-based biosensor for the detection of atrazine," Analytical Letters, 37,1671-1681, 2004.

10. I. Jung, and Y.Roh, "Design and fabrication of piezoceramic bimorph vibration sensors," Sensors and Actuators A, 69, pp. 259-266, 1998.

11. G.M.Spinks, D.Z.Zhou, L.Liu, and G.G. Wallace, "The amounts per cycle of polypyrrole electromechanical actuators," Smart Mater. \& Struct., 12, pp. 468-472, 2003.

12. G.Spinks, L.B.Zhao, W.H.Li, Y.Z.Wu, G.Z.Zhou and G.G. Wallace, "Synthesis, modeling and characterization of conducting polymers", SPIE Symposium on Smart Materials, Nano- and Micro-Smart Systems, Sydney, 12-15 December, 2004, Paper No: 5648-28. 